\title{
BNL ACCELERATOR TEST FACILITY CONTROL SYSTEM UPGRADE
}

\author{
R. Malone, I. Ben-Zvi, X. Wang, V. Yakimenko \\ $\mathrm{BNL}^{\dagger}$, Upton, NY 11973, USA
}

\begin{abstract}
Brookhaven National Laboratory's Accelerator Test Facility (ATF) has embarked on a complete upgrade of its decade old computer system. The planned improvements affect every major component: processors (Intel Pentium replaces VAXes), operating system (Linux/Real-Time Linux supplants OpenVMS), and data acquisition equipment (fast Ethernet equipment replaces CAMAC serial highway.) This paper summarizes the strategies and progress of the upgrade along with plans for future expansion.
\end{abstract}

\section{INTRODUCTION}

Commissioned in 1990, ATF was formed to carry out long-term research in accelerator physics including new concepts, devices and technologies to improve future linear colliders and light sources. As a dedicated user facility, around 1100 hours per year of beam time are made available to researchers from government laboratories, universities and industry to perform committee-approved experiments at the facility's three beam lines. ATF's core capabilities include a high brightness photoinjector electron gun, a $70 \mathrm{MeV}$ electron accelerator and a fully instrumented experiment hall. Facility operators and users have access to essentially all of this equipment through the main control system.

\section{OVERVIEW OF PRESENT SYSTEM}

The ATF control system hardware (show schematically in Fig. 1) was originally configured and installed in 1990 [1] and has remained essentially unchanged since that time.

Centered around a $114 \mathrm{MHz}$ VAX 4200 (single CPU) equipped with $56 \mathrm{Mb}$ of main memory, $1.7 \mathrm{~Gb}$ of disk storage and $10 \mathrm{MHz}$ Ethernet interface, the Q-bus based VAX has provided control system and data acquisition services continuously for over a decade. Data acquisition is CAMAC-based, driven by a Kinetic Systems Corporation model 2160 serial highway driver [2] with six crates positioned at various locations around the facility. Each is equipped with a type L2 enhanced serial crate controller, communicating over a $5 \mathrm{MHz}$ metallic serial highway cable, which is daisy-chained between the crates.

The software consists of Compaq (originally Digital)'s Open VMS operating system [3] together with TGV's

Supported by the U.S. Department of Energy under contract DE-AC02-98CH10886

Multinet TCP/IP stack. Compaq compilers were used to develop application programs in $\mathrm{C}$ and Fortran. All operator displays and supporting databases were built using Vsystem, a commercial software tool package from Vista Control Systems, Inc. [4]

Figure 1: Schematic of present ATF control system.

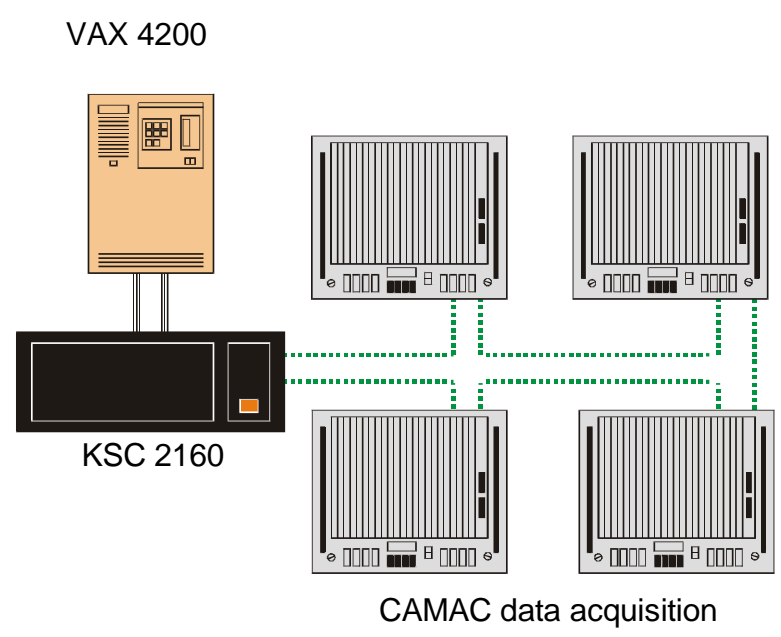

Although the top-level functionality of the control system is highly sophisticated and readily applicable to the required tasks, the limitations of decade-old hardware are apparent. The primary problem is the speed of the system: activation of new processes is slow, bordering on the very edge of acceptability. The number of applications programs and services developed since commissioning has increased to the point where the severe limitation of computing resources makes it impossible to add new advanced capabilities such as extended on-line modeling and global feedback algorithms. The support of legacy hardware and software is also an issue for concern. The need to improve the system has been apparent since 1995, but limited funding precluded any upgrade until recently.

\section{UPGRADE PHILOSOPHY AND KEY DECISIONS}

Early in the discussions of how best to improve the control system, a clear consensus on general strategies emerged:

- Use off-the-shelf, commodity hardware and software wherever possible.

- Invest in those technologies with clear expansion and upgrade pathways and which improve ATF's overall flexibility, stability and reliability.

- Preserve investments made in data acquisition and control hardware while allowing for new hardware families to be introduced (VME, VXI.) 
- Maintain the current level of familiarity users have with the present system by porting applications software and operator displays, hopefully making the behind-the-scenes upgrades transparent. Any plan that would require major retraining of personnel was deprecated.

But the most important (and challenging) requirement:

- Minimize disruption to the on-going scientific program. All upgrade work must occur while still maintaining the present control system at full operational readiness. Final cutover to the new system should entail as little disturbance to operations as practicable.

Based on these guidelines, key decisions were reached:

- Move from VAX hardware to the ubiquitous Intel Pentium-based machines (see specifications below.)

- Replace L2-serial crate controllers with $100 \mathrm{MHz}$ Ethernet crate controllers, fed through a fast Ethernet switch driven from the new host.

- Remove the serial highway, replacing it with a private fast Ethernet sub-network.

- Use Linux instead of VMS, employing real-time Linux (RT-Linux [5]) for hard real-time tasks. (Windows NT was not found to be sufficiently stable in meeting ATF requirements during preliminary evaluations.)

- Replace operator workstations (previously XWindow terminals) with Windows NT PCs. This allows X-displays to operate side-by-side with "shrink-wrapped" applications like Mathcad, Matlab and LabView.

- Maintain external networked services as is, instead rewriting only the network socket listener which supports such services. (This includes video frame grabbers, Mathcad interfaced via network, visual $\mathrm{C}++$ applications and LabView programs.)

- Continue with the successful use of Vsystem development tools

The machine chosen to be the new control system host is an IBM Netfinity $8500 \mathrm{R}$ server [6]. Initially, it will operate with two $700 \mathrm{MHz}$ Pentium-III Xeon processors (expandable to eight CPUs), $1.5 \mathrm{~Gb}$ main memory (expandable to $64 \mathrm{~Gb}$ ), 17Gb RAID disk storage and 40Gb SCSI DLT tape drive. Three network interface cards permit external communications: one strictly for data acquisition, the second for data communication within ATF and the third for general network access. The machine is also equipped with three redundant, hot-swap power supplies, fed from an uninterruptible power supply with sufficient capacity to operate for around 30 minutes in the event of $\mathrm{AC}$ power loss. The new host and data acquisition is shown schematically in Figure 2.
Figure 2: Schematic of new ATF control system

\section{THE SYSTEM IN TRANSITION}

IBM Netfinity $8500 R$

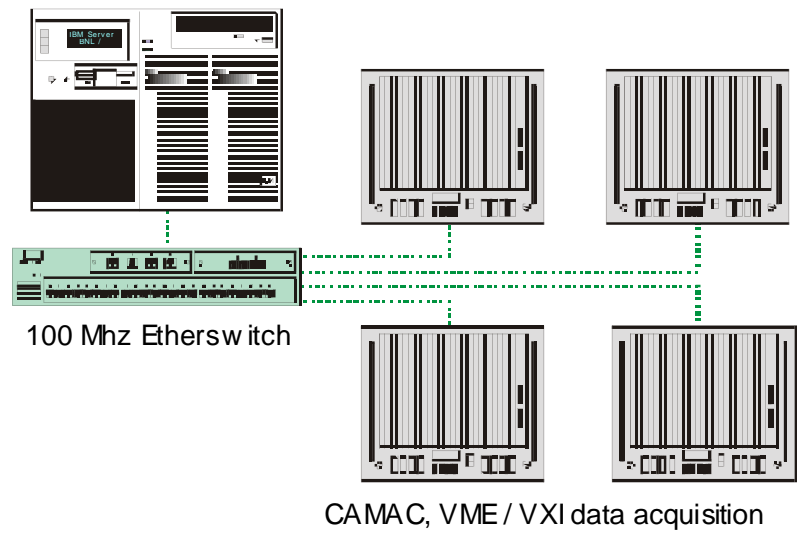

\subsection{Parallel operation of data acquisition hardware}

During the upgrade process, each CAMAC crate will continue to operate with its L2 controller acting as a primary controller with the new Ethernet crate controllers introduced as auxiliary controllers. This will permit shared access to the CAMAC hardware for both the old and new control systems. This allows testing of the new system with actual loads during maintenance periods, nights and weekends and other times when regular beam operation is not required. It also hastens porting of existing software and provides a degree of quality control since anomalous behavior of new applications can be understood by rapid and direct comparison to a known working system. When all upgrade and porting tasks have been completed, the serial controllers and the serial highway will be removed and the Ethernet controllers reconfigured for primary controller operation. At that point, the VAX will be removed from service and the entire operation will be through the new IBM host.

\subsection{Graphic User Interface}

One of the important features of the control system is its graphical user interface, comprised of around 800 displays that present various views of the accelerator, from high-level overviews of entire beam lines, down to individual devices. (Figure 3 shows a sample screen dump from an operator terminal.) Facility operators and visiting users have come to appreciate the user-friendliness inherent in the displays and have built up significant institutional knowledge of "where to find things" using them. To maintain the same level of user productivity, it is critical therefore to maintain the same look and feel of the present control system once it is fully operational on the new hardware. 


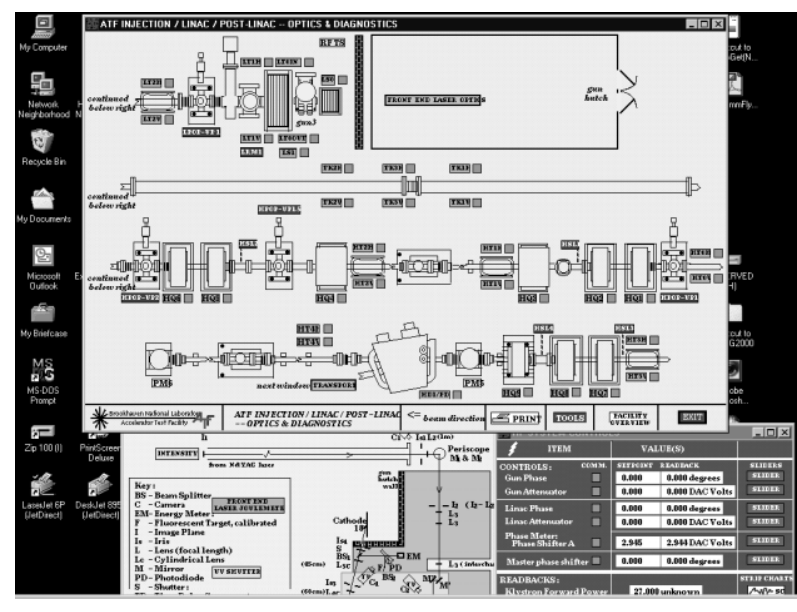

Figure 3: Sample Operator Display

It would be far too difficult (and error prone) to manually reconstruct all of the displays of the existing system. To automate the conversion process, a two-pass approach is used. First, using a Vsystem conversion utility, the static drawing elements (lines, geometric figures, text headings, etc.) are translated from the VMS metafile format to that needed under Linux.

At this point, the displays appear to be correct, but still have internal problems, mostly related to VMS filename syntax and upper/lower case issues. In the second step, a perl script analyzes the file references in each drawing, converting them to appropriate Linux filenames A generalization of the perl script also analyzes the entire set of displays, looking for references to non-existent displays or possible orphans (displays not referenced by any other.) Such tools are vital in ensuring the proper operation of the conversion process and can be also be reused in the future as new displays are created and added to the active system.

\subsection{Applications program porting}

Another area of significant effort is in porting applications programs. Some 75,000 lines of code have been generated over the past 10 years, most all of it needed for daily operations. Originally written in $\mathrm{C}$ and Fortran, these applications are being rewritten in $\mathrm{C}++$. This presents the largest challenge to the entire upgrade task and is its most labor-intensive. Development tools from the Portland Group, Inc. will be used since they can exploit the multiple processors of the new system and also handle VAX compiler language extensions. Again, perl scripts will be used in the conversion process by generating templates for new source code which is then manually edited to translate from the original.

\section{PROGRESS AND PLANS}

To date, the new IBM server has been delivered, Linux installed and configured. The private section of fast Ethernet has been extended to all areas of the facility requiring data acquisition and the switch installed. Fiber optic cable has also been installed at all key locations in preparation for future expansion. All of the operator displays have been converted to Linux file format and the supporting databases have been tested. The CAMAC equipment has been configured for dual-controller operations and will be exercised heavily during Summer 2001.Work continues porting applications programs and will continue into Winter 2002, hopefully when the present system will be retired and the new one will assume full operational status.

Future plans call for new hardware families (VME, VXI) to be introduced to provide a wider spectrum of data acquisition hardware beyond the limited offerings of CAMAC.

With additional processing power available, more extensive on-line modeling will be initiated as well as development and implementation of new feedback and control algorithms.

\section{CONCLUSIONS}

Brookhaven's Accelerator Test Facility is undertaking an aggressive campaign to upgrade its computer control system. A careful, cost-effective plan is being executed which will enhance the facility's capabilities and provide better service to the accelerator physics community. Users can look forward to significantly improved performance, without the need for retraining. Work on the conversion effort is expected to be concluded around Winter 2002.

\section{REFERENCES}

[1] R. Malone, I. Bottke, R. Fernow, and I. Ben-Zvi, "The BNL Accelerator Test Facility Control System", Proceedings of the Eighth IEEE Real-time Conference, Vancouver, B.C., June 8-11, 1993 (TRIUMF report TRI-93-1)

[2] Kinetic Systems Corporation, 900 North State Street, Lockport, IL 60441

[3] Compaq Computer Corporation, PO Box 69200, Houston, TX 77269

[4] Vista Control Systems, 176 Central Park Square, Los Alamos, NM 87544

[5] FSM Labs, PO Box 1822, Socorro, NM 87801

[6] IBM Corporation, New Orchard Road, Armonk, NY 10504 